\title{
INDEX TO VOLUME 12
}

Agboola, Adebisi, and Howard, Benjamin Anticyclotomic Iwasawa the-

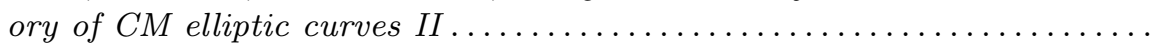

Akaho, Manabu Intersection theory for Lagrangian immersions .......... Alvarez-Montaner, Josep, Blickle, Manuel, and Lyubeznik, Gennady Generators of $D-$ modules in positive characteristic ...............

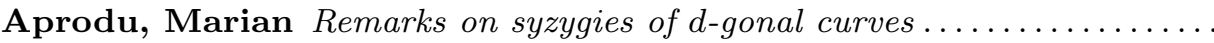

Arinkin, D. On $\lambda$-connections on a curve where $\lambda$ is a formal parameter . .

Avramov, Luchezar L., and Iyengar, Srikanth Gaps in Hochschild cohomology imply smoothness for commutative algebras .................

Bennett, Jonathan, Carbery, Anthony, and Wright, James A nonlinear generalisation of the Loomis-Whitney inequality and applications .

Bini, Gilberto Chern Classes of the Moduli Stack of Curves..............

Blickle, Manuel see Alvarez-Montaner, Josep, Blickle, Manuel, and

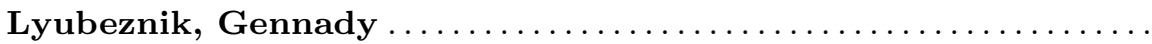

Boutet de Monvel, L. Logarithmic trace of toeplitz projectors ...........

Boylan, Matthew 2-adic properties of Hecke traces of singular moduli.....

Buch, Anders Skovsted, Sottile, Frank, and Yong, Alexander Quiver

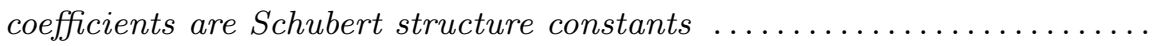

Buchweitz, Ragnar-Olaf, Green, Edward L., Madsen, Dag, and Solberg, Øyvind Finite Hochschild cohomology without finite global dimen-

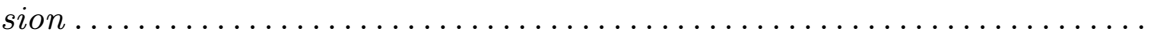

Carbery, Anthony see Bennett, Jonathan, Carbery, Anthony, and

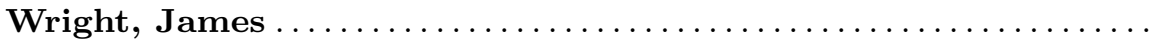

Charney, Ruth, and Crisp, John Automorphism groups of some affine and

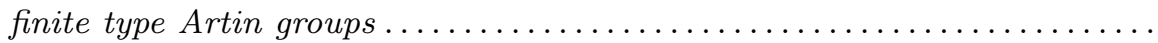

Chau, Albert, and Tam, Luen-Fai $A$ note on the uniformization of gradient

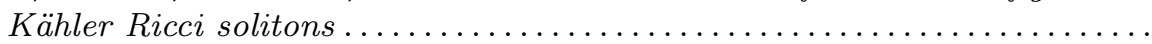

Chen, PoNing, and Dolgushev, Vasiliy A simple algebraic proof of the

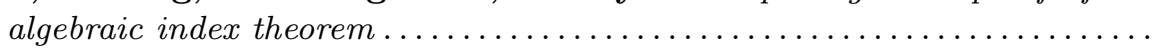

Christiansen, T., and Hislop, P. D. The resonance counting function for Schrödinger operators with generic potentials .................

Colliander, J., Raynor, S., Sulem, C., and Wright, J. D. Ground state mass concentration in the $L^{2}$-critical nonlinear Schrödinger equation below

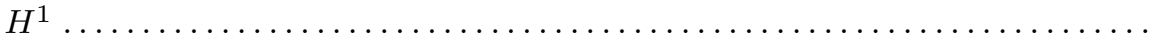

Corn, Patrick Del Pezzo surfaces of degree $6 \ldots \ldots \ldots \ldots \ldots \ldots \ldots \ldots \ldots \ldots$

Corti, Alessio, and Smith, Ivan Conifold transitions and Mori theory...

Crisp, John see Charney, Ruth, and Crisp, John ...............

Dairbekov, Nurlan S., and Paternain, Gabriel P. Longitudinal KAMcocycles and action spectra of magnetic flows ..................

Damanik, David, and Killip, Rowan Ergodic potentials with a discontin-

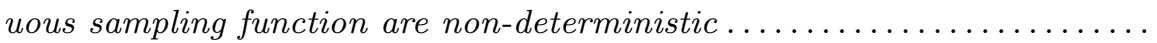

Dani, S.G., and Schmidt, Klaus Addendum to the paperAffinely infinitely divisible distributions and the embedding problem ................

611

543

459

387

551

789

443

759

459

401

593

567

805

443

321

821

357

75

767

321

719

187

817 
Đoković, Dragomir Ž., and Zhao, Kaiming Jordan decomposition of bi-

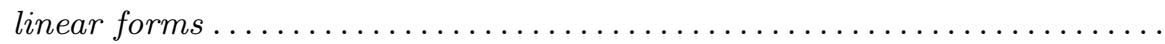

Dolgushev, Vasiliy see Chen, PoNing, and Dolgushev, Vasiliy ......

Etingof, P., and Graña, M. Quantization of non-unitary geometric classical

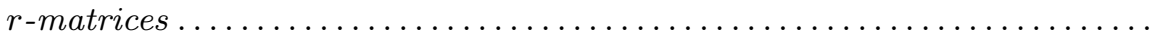

Fang, Xiang The Fredholm index of quotient Hilbert modules . . . . . . . . . .

Farb, Benson, and Ivanov, Nikolai V. The Torelli geometry and its ap-

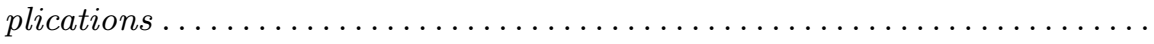

Franc Forstnerič, and Winkelmann, Jörg Holomorphic discs with dense

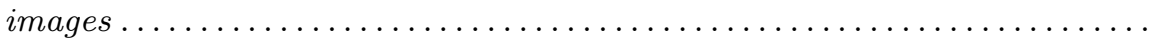

Frances, Charles, and Zeghib, Abdelghani Some remarks on conformal pseudo-Riemannian actions of simple Lie groups ...................

Galatolo, Stefano Dimension via waiting time and recurrence...........

Gan, Wee Liang On a theorem of Conant-Vogtmann .................

Goldman, Wiliam M., and Neumann, Walter D. Homological action of the modular group on some cubic moduli spaces ....................

Graña, M. see Etingof, P., and Graña, M. ................

Green, Edward L. see Buchweitz, Ragnar-Olaf, Green, Edward L.,

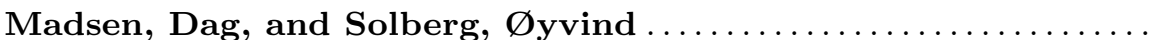

Greenblatt, Michael Stability of sublevel set estimates and sharp $L^{2}$ regu-

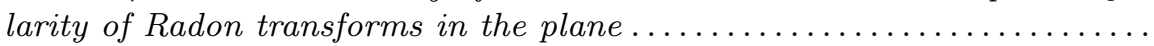

Grushevsky, Samuel, and Manni, Riccardo Salvati Two generalizations

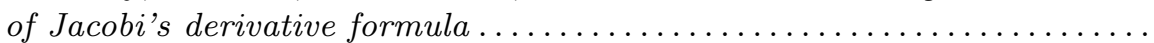

Guillarmou, Colin Resonances and scattering poles on asymptotically hyperbolic manifolds ..................................

Hacon, Christopher D., and Pardini, Rita Birational characterization of

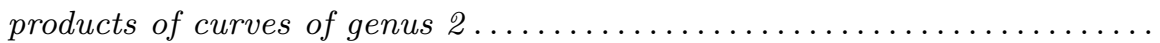

Hain, Richard M. see Kim, Minhyong, and Hain, Richard M. .....

Hencl, Stanislav, Koskela, Pekka, and Onninen, Jani A note on ex-

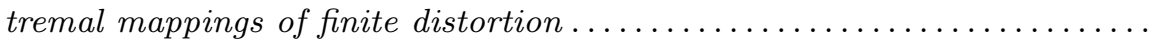

Hislop, P. D. see Christiansen, T., and Hislop, P. D. . . . . . . 821

Horozov, I. Euler characteristics of arithmetic groups . . . . . . . . . . 275

Howard, Benjamin see Agboola, Adebisi, and Howard, Benjamin . . . 611

Ionel, Marianty, Karigiannis, Spiro, and Min-Oo, Maung Bundle constructions of calibrated submanifolds in $\mathbb{R}^{7}$ and $\mathbb{R}^{8} \ldots \ldots \ldots \ldots \ldots \ldots$

Ionescu, Alexandru D., and Kenig, Carlos E. Well-posedness and local

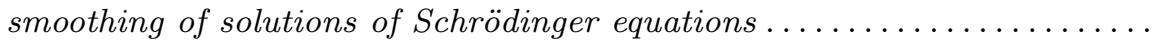

Ito, Toshikazu, and Scárdua, Bruno On the non-existence of a codimension one holomorphic foliation transverse to a sphere ................

Ivanov, Nikolai V. see Farb, Benson, and Ivanov, Nikolai V. ........ Iyengar, Srikanth see Avramov, Luchezar L., and Iyengar, Srikanth

Jost, Jürgen, and Yang, Yi-Hu Heat flow for horizontal harmonic maps

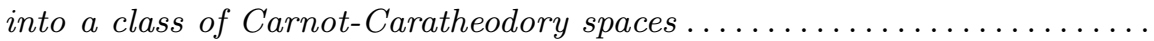

Karigiannis, Spiro see Ionel, Marianty, Karigiannis, Spiro, and Min-

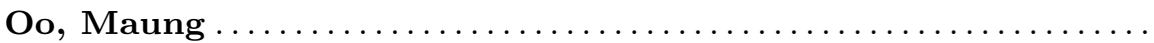

Kashaev, R. M. Coordinates for the moduli space of flat $P S L(2, \mathbb{R})$

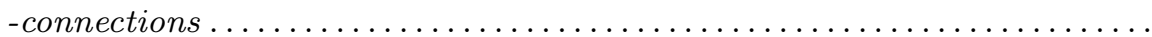

Kato, Jun Existence and uniqueness of the solution to the modified Schrödinger

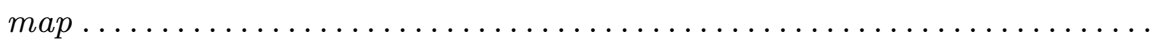

341

655

141

911

293

265

49

377

749

575

141

805

1

921

103

129

155

231

493

193

713

293

789

513

493 
Kedlaya, Kiran S. Frobenius modules and de Jong's theorem ........... 303

Kenig, Carlos E. see Ionescu, Alexandru D., and Kenig, Carlos E. . 193

Khare, Chandrashekhar, Larsen, Michael, and Ramakrishna, Ravi Transcendental $\ell$-adic Galois representations ................ 685

Killip, Rowan see Damanik, David, and Killip, Rowan . . . . . . . 187

Kim, Minhyong, and Hain, Richard M. The Hyodo-Kato theorem for

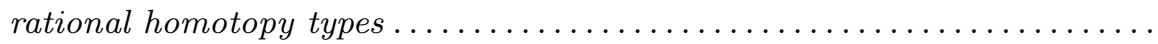

Kokotov, Alexey, and Strachan, Ian A. B. On the isomonodromic taufunction for the Hurwitz spaces of branched coverings of genus zero and

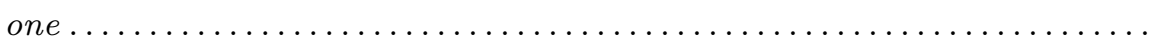

Kolář Martin Normal forms for hypersurfaces of finite type in $\mathbb{C}^{2} \ldots . . .$.

Korkmaz, Mustafa On stable torsion length of a Dehn twist............

Korman, Philip, Li, Yi, and Ouyang, Tiancheng Computing the location

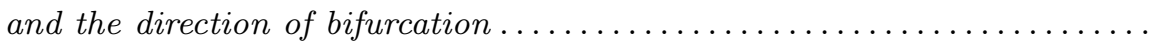

Koskela, Pekka see Hencl, Stanislav, Koskela, Pekka, and Onninen,

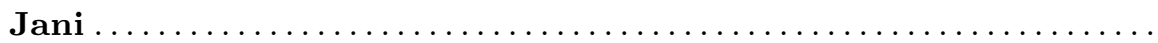

Lanzani, Loredana, and Stein, Elias M. A note on div curl inequalities .

Larsen, Michael see Khare, Chandrashekhar, Larsen, Michael, and

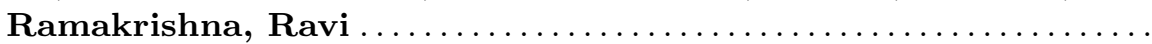

Li, Yi see Korman, Philip, Li, Yi, and Ouyang, Tiancheng ......... Lyubeznik, Gennady see Alvarez-Montaner, Josep, Blickle, Manuel,

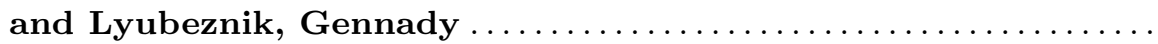

Madsen, Dag see Buchweitz, Ragnar-Olaf, Green, Edward L., Madsen, Dag, and Solberg, $\varnothing_{y v i n d ~} \ldots \ldots \ldots \ldots \ldots \ldots \ldots \ldots \ldots \ldots$

Manni, Riccardo Salvati see Grushevsky, Samuel, and Manni, Ric-

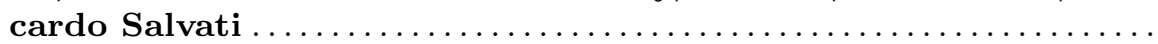

Matsuki, Kenji, and Olsson, Martin Kawamata-Viehweg vanishing as

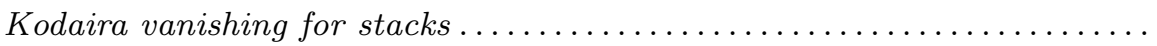

Migliore, Juan, Nagel, Uwe, and Römer, Tim The multiplicity conjecture

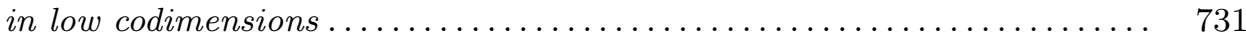

Mijatović, Aleksandar Simplical structures of knot complements ....... 843

Miller, Luc Unique continuation estimates for the Laplacian and the heat

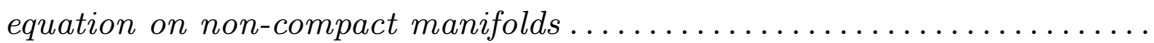

Min-Oo, Maung see Ionel, Marianty, Karigiannis, Spiro, and Min-Oo,

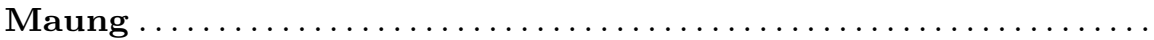

Nagel, Uwe see Migliore, Juan, Nagel, Uwe, and Römer, Tim ......

Naumann, N. A quantitative sharpening of Moriwaki's arithmetic Bogomolov

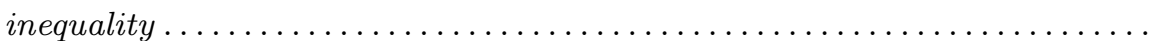

Neumann, Walter D. see Goldman, Wiliam M., and Neumann, Wal-

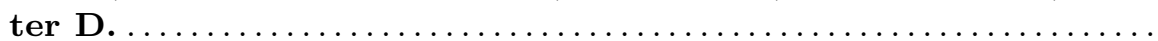

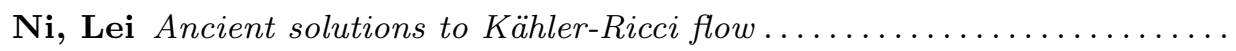

Nicoleau, François An inverse scattering problem for short-range systems

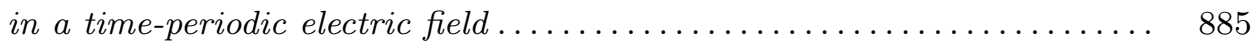

Olsson, Martin see Matsuki, Kenji, and Olsson, Martin ......... 207

Onninen, Jani see Hencl, Stanislav, Koskela, Pekka, and Onninen,

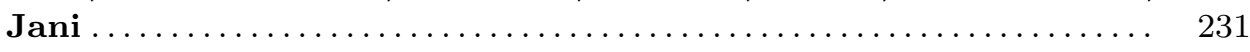

Osserman, Brian Deformations of covers, Brill-Noether theory, and wild

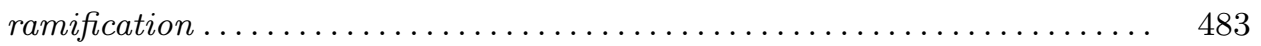

Ouyang, Tiancheng see Korman, Philip, Li, Yi, and Ouyang, Tiancheng 933 
Pardini, Rita see Hacon, Christopher D., and Pardini, Rita .......

Park, Jongil, Stipsicz, András I., and Szabó, Zoltán Exotic smooth

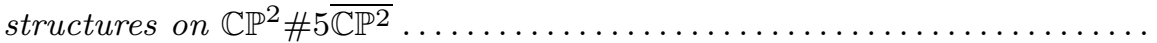

Paternain, Gabriel P. see Dairbekov, Nurlan S., and Paternain,

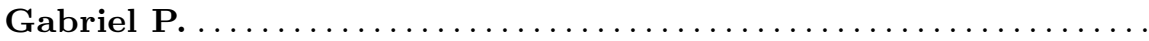

Polini, Claudia, Ulrich, Bernd, and Vasconcelos, Wolmer V. Normalization of ideals and Briançon-Skoda numbers ................ 827

Polishchuk, A. Noncommutative Proj and coherent algebras ........... 63

Poonen, Bjorn Unramified covers of Galois covers of low genus curves . . . 475

Prasad, Gopal, and Rapinchuk, Andrei S. Zariski-dense subgroups and transcendental number theory ........................ 239

Römer, Tim see Migliore, Juan, Nagel, Uwe, and Römer, Tim . . . . 731

Ramakrishna, Ravi see Khare, Chandrashekhar, Larsen, Michael,

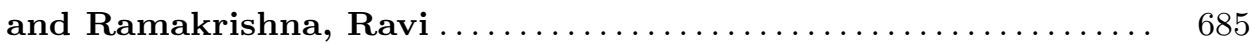

Rapinchuk, Andrei S. see Prasad, Gopal, and Rapinchuk, Andrei S. 239

Raynor, S. see Colliander, J., Raynor, S., Sulem, C., and Wright, J.

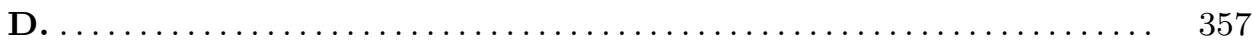

Sadovskaya, Victoria On uniformly quasiconformal anosov systems . . . . 425

Scárdua, Bruno see Ito, Toshikazu, and Scárdua, Bruno . . . . . . . . . 713

Schenker, Jeffrey H., and Schulz-Baldes, Hermann Semicircle law and freeness for random matrices with symmetries or correlations ..........

Schlenker, Jean-Marc Hyperideal circle patterns .................

Schmidt, Klaus see Dani, S.G., and Schmidt, Klaus ............

Schulz-Baldes, Hermann see Schenker, Jeffrey H., and Schulz-Baldes,

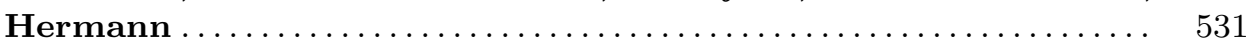

Sesum, Natasa Convergence of a Kähler-Ricci flow ................... 623

Skorobogatov, Alexei Shimura coverings of Shimura curves and the Manin

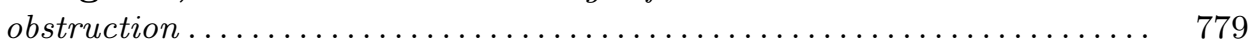

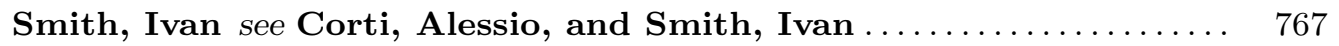

Solberg, Øyvind see Buchweitz, Ragnar-Olaf, Green, Edward L., Madsen, Dag, and Solberg, $\varnothing_{y v i n d} \ldots \ldots \ldots \ldots \ldots \ldots \ldots \ldots \ldots \ldots \ldots \ldots \ldots \ldots$

Sottile, Frank see Buch, Anders Skovsted, Sottile, Frank, and Yong,

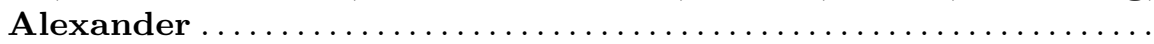

Stein, Elias M. see Lanzani, Loredana, and Stein, Elias M. .......

Stipsicz, András I. see Park, Jongil, Stipsicz, András I., and Szabó,

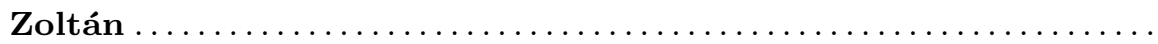

Strachan, Ian A. B. see Kokotov, Alexey, and Strachan, Ian A. B. .

Strichartz, Robert S. Laplacians on fractals with spectral gaps have nicer

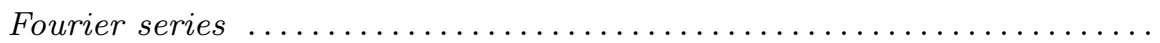

Sulem, C. see Colliander, J., Raynor, S., Sulem, C., and Wright, J.

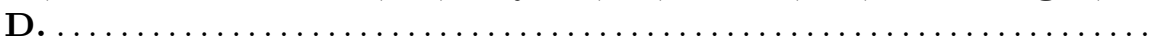

Szabó, Zoltán see Park, Jongil, Stipsicz, András I., and Szabó, Zoltán Tam, Luen-Fai see Chau, Albert, and Tam, Luen-Fai ............ Tao, Terence An uncertainty principle for cyclic groups of prime order.... Ulrich, Bernd see Polini, Claudia, Ulrich, Bernd, and Vasconcelos,

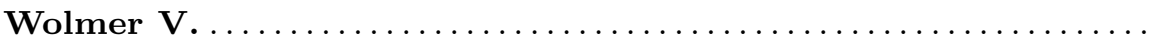

Vasconcelos, Wolmer V. see Polini, Claudia, Ulrich, Bernd, and Vasconcelos, Wolmer V. 
Vasy, András, and Wunsch, Jared Absence of super-exponentially decaying eigenfunctions on Riemannian manifolds with pinched negative curvature

Verbitsky, Misha Stable bundles on positive principal elliptic fibrations....

Winkelmann, Jörg see Franc Forstnerič, and Winkelmann, Jörg .....

Wright, J. D. see Colliander, J., Raynor, S., Sulem, C., and Wright,

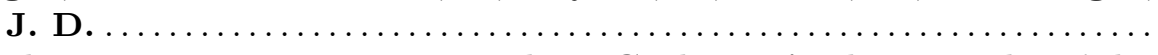

Wright, James see Bennett, Jonathan, Carbery, Anthony, and Wright,

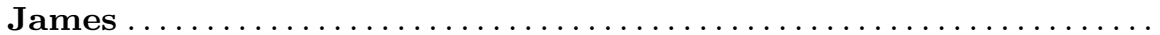

Wunsch, Jared see Vasy, András, and Wunsch, Jared ................ 643

Yan, Yu The existence of horizons in an asymptotically flat 3-manifold 219

Yang, Yi-Hu see Jost, Jürgen, and Yang, Yi-Hu .................. 513

Yong, Alexander see Buch, Anders Skovsted, Sottile, Frank, and Yong, Alexander ................................. 567

Zeghib, Abdelghani see Frances, Charles, and Zeghib, Abdelghani .. 49

Zhang, Qiao On the cubic moment of quadratic dirichlet L-functions . . . . . 413

Zhao, Kaiming see Đoković, Dragomir Ž., and Zhao, Kaiming ..... 341 